\title{
The contribution of geomagnetic observatories and magnetic models to the study of secular variation and jerks in Antarctica
}

\author{
A. Meloni, L. Cafarella, P. De Michelis, and R. Tozzi \\ Istituto Nazionale di Geofisica e Vulcanologia,Via di Vigna Murata 605, 00143, Rome, Italy, (meloni@ingv.it)
}

\begin{abstract}
Some of the most interesting features of the geomagnetic field and its time variations are displayed in polar areas. Observatory monthly means usually provide an excellent opportunity to study the temporal changes of the magnetic field at a given location. Unfortunately, on the Antarctic continent the distribution of the permanent groundbased observatories does not permit a uniform coverage of the examined area. Furthermore, the magnetic records are characterized by intense external disturbances and noise that make the analysis of the magnetic field difficult. To improve our knowledge of the secular variation and detect the presence of secular variation impulses (geomagnetic jerks) in Antarctica, we use both observatory data and the CM4 quiet time magnetic field model. In particular CM4 improves our knowledge of geomagnetic jerks over Antarctica through the study of the sign changes of the secular acceleration maps.
\end{abstract}

Citation: Meloni, A., L. Cafarella, P. De Michelis, and R. Tozzi (2007), The contribution of geomagnetic observatories and magnetic models to the study of secular variation jerks in Antarctica, in Antarctica: A Keystone in a Changing World - Online Proceedings of the $10^{\text {th }}$ ISAES, edited by A. K. Cooper and C. R. Raymond et al., USGS Open-File Report 2007-1047, Short Research Paper 071, 5 p.; doi:10.3133/of2007-1047.srp071

\section{The geomagnetic observatories}

The magnetic field observed at the Earth's surface is mainly a manifestation of a magnetohydrodynamic dynamo acting in the interior of our planet. The field, caused by electric currents flowing in the Earth's fluid outer core, is known as the core field and it represents over 97 per cent of what is observed at the Earth's surface. In addition to sources in the Earth's core, the geomagnetic field is produced by sources in the lithosphere and by contributions external to the Earth caused by electric currents flowing in the ionosphere, magnetosphere, as well as from the coupling of these currents and the currents flowing between the two Earth's hemispheres.

Geomagnetic observatories, where magnetic elements are regularly and continuously recorded, monitor the geomagnetic field. Geomagnetic observatories measure the total intensity $\mathrm{F}$ of the field and the three Cartesian components indicating respectively the north-south $(X)$, the west-east $(Y)$ and the vertical $(Z)$ intensities. To facilitate data circulation, the scientific community of magnetic data users has launched an international coordinated program called INTERMAGNET adopting modern and strict standards for the measuring and recording equipment (Kerridge, 2001, see also http://www.intermagnet.org/).

The global distribution of geomagnetic observatories is strongly unbalanced in favor of the Northern Hemisphere and leaves the Southern Hemisphere poorly covered owing to its scant land coverage. Unfortunately, most of Antarctic geomagnetic observatories have been operating for only a few years. Consequently, long continuous time series of the geomagnetic field in Antarctica can be provided by about only ten observatories. Most of them are located along the coast for practical and historical reasons. Thus, Antarctic magnetic data are influenced by coastal effects and crustal field contamination. A more useful observatory network would cover the entire continent uniformly, which is a very difficult task in Antarctica. Particularly important installations include the South Pole and Vostok stations that have ensured continuous geomagnetic records over the past 50 years. In 2004 at Dome C, a geomagnetic observatory was installed at Concordia station (IAGA code DMC), as the result of an agreement between the French (IPEV) and Italian Antarctic Programs (PNRA) (e.g. Lepidi et al, 2003).

The main importance of the ensemble of world magnetic observatories, including those in Antarctica, is therefore the capability of continuously monitoring the geomagnetic field.

\section{Secular variation and geomagnetic jerks in Antarctica}

The term secular variation identifies slow changes of the Earth's magnetic field on time scales that range from a few years to millennia. Secular variation, like the magnetic field itself, originates from the core fluid flows by which the field is generated. The examination of secular variation in Antarctic observatory data in the last five decades shows a rapid decrease in the total magnetic field (e.g. Rajaram et al., 2002). Some investigators (e.g. De Santis et al., 2004) have speculated that this rapid decrease would be of global relevance implying the development of a dipole reversal as has happened several times in the Earth's history, but others have denied this possibility (Gubbins et al., 2006).

Although secular variation usually occurs as a smooth change of the main field with time, episodes of abrupt changes known as geomagnetic jerks (Courtillot et al., 1978) have occurred in the past. They are manifested in the magnetic record as sudden $\mathrm{V}$-shaped changes in the slope of secular variation, that are impulses or Delta functions in the third time derivative of the field. In reality, the duration of a jerk may be from several months to a few years. Furthermore, several studies suggest that 
geomagnetic jerks show a time lag of a few years between the two hemispheres (Alexandrescu et al., 1996; De Michelis et al., 2000; De Michelis and Tozzi, 2005). In spite of the large amount of work done, little is understood about the physical origin of this phenomenon. Waddington et al. (1995) suggested that geomagnetic jerks could be induced by sudden accelerations of the metallic fluid core and consequently they could be related to global changes in the flow near the core surface. Taking this suggestion into account, Bloxham et al. (2002) showed that jerks can be explained in terms of a combination of a steady flow and a simple time-varying axisymmetric, equatorially symmetric, toroidal zonal flow, which is consistent with torsional oscillations. The internal origin of this phenomenon, which has been established through both spherical harmonics (Malin and Hodder, 1982) and wavelet analyses (Alexandrescu et al., 1996), implies that this event is a key factor for understanding the Earth's internal dynamics and that it is also an excellent tool to constrain lower mantle conductivity (Alexandrescu et al., 1996) and its possible lateral heterogeneity (Nagao et al., 2003). Magnetic records show that jerks occurred around 1901, 1913, 1925, 1932, 1949, 1969, 1978 and 1991 (Alexandrescu et al., 1996; Macmillan, 1996). Three of these jerks (1969, 1978 and 1991) were unquestionably of global extension, three (1901, 1913 and 1925) may have been of similar extension, while the other two (1932 and 1949) were not seen everywhere at the Earth's surface. Recently, Mandea et al., (2000) pointed out another jerk in 1999 from analysing the series of magnetic measurements at some European observatories, and an additional one in 2003 was detected in satellite data (Olsen and Mandea, 2007).

In the past, different types of analysis have been proposed to identify jerks in the trend of geomagnetic secular variation. The main limit of these classical methods is that they assume the existence of an impulse in the secular variation, and its date is found, for example, by inspection of the time series or by finding the date that minimises the root-mean-square residual to straight-line fits on the secular variation. To achieve a systematic study of jerks, without any a priori assumption on their existence, location and form, Alexandrescu et al. (1996) applied wavelet analysis to the monthly mean geomagnetic time series. Subsequently, De Michelis and Tozzi (2005) improved this method of analysis by introducing the local intermittency measure approach to the detection of these events.

A serious limitation regarding the investigation of these internal processes with time-scales of months to a few years is the effect of geomagnetic variations of external origin, because they contribute significantly on these time-scales. This is the reason why jerks were initially studied using annual mean values of the magnetic field. However, with the use of monthly mean values of the geomagnetic field, different models and techniques have been applied to remove the external contributions and the random noise from the geomagnetic field data. However, these methods are critically affected by the length, continuity, and quality of the magnetic records. There are regions on the Earth's surface where it is difficult to find several observatories satisfying this condition. This is the case of Antarctica where the number of observatories is limited in comparison with the extension of the continent and where the intensive external disturbances and noise make the classical analysis of geomagnetic jerks difficult. In the following, we study the geomagnetic jerks over Antarctic region using a new approach based on the comprehensive geomagnetic field model CM4 (Sabaka et al., 2004) and secular acceleration maps. Recently, Chambodut and Mandea (2005) have used this model for a global search of geomagnetic jerks. They have applied CM4 to evaluate the secular variation trend on the Earth's surface and, using the classical method of straight-line fit, they have determined the time occurrences of the last three global jerks of the 20th century. However, they have not been able to find conclusive results for the Antarctic region. To overcome this problem, we have studied the secular acceleration maps of the geomagnetic field and detected jerks as jumps in the secular acceleration values. A similar approach has been used by Chambodut et al. (2005) but details are not available.

\section{Using the CM4 magnetic field model}

The spatial and the temporal scales of some of the contributions to the Earth's magnetic field overlap, making it difficult to separate their effects from samples of the observed field. A very successful approach known as "comprehensive modeling" has been developed to overcome this problem. The Comprehensive Models (CMs) are derived from observatory data as well as data from magnetic satellite missions. The last published model, denoted CM4 (Sabaka et al., 2004) is an extension of CM3 that includes not only POGO and Magsat satellite data, but also scalar data from the Ørsted and CHAMP satellites. The resulting model shows great improvement over its predecessors in terms of completeness of sources, time span, and the noise reduction in the recovered parameters. This model attempts not only to separate the internal from the external signals, even those induced from the core, during quiet-time conditions, but also provides a global description of the field's evolution through time.

We think that CM4 can also be particularly useful in the study of secular variation over the Antarctic region where a sufficient number of observatories is lacking. Indeed, a serious limitation regarding the investigation of the internal processes with time-scales of months to a few years is the corrupting effect of geomagnetic variations of external origin, that contribute significantly to shorter secular variations. Here, the comprehensive modeling approach can help us in the study of geomagnetic jerks, especially where the external disturbances are more intense as in the Antarctic region. 

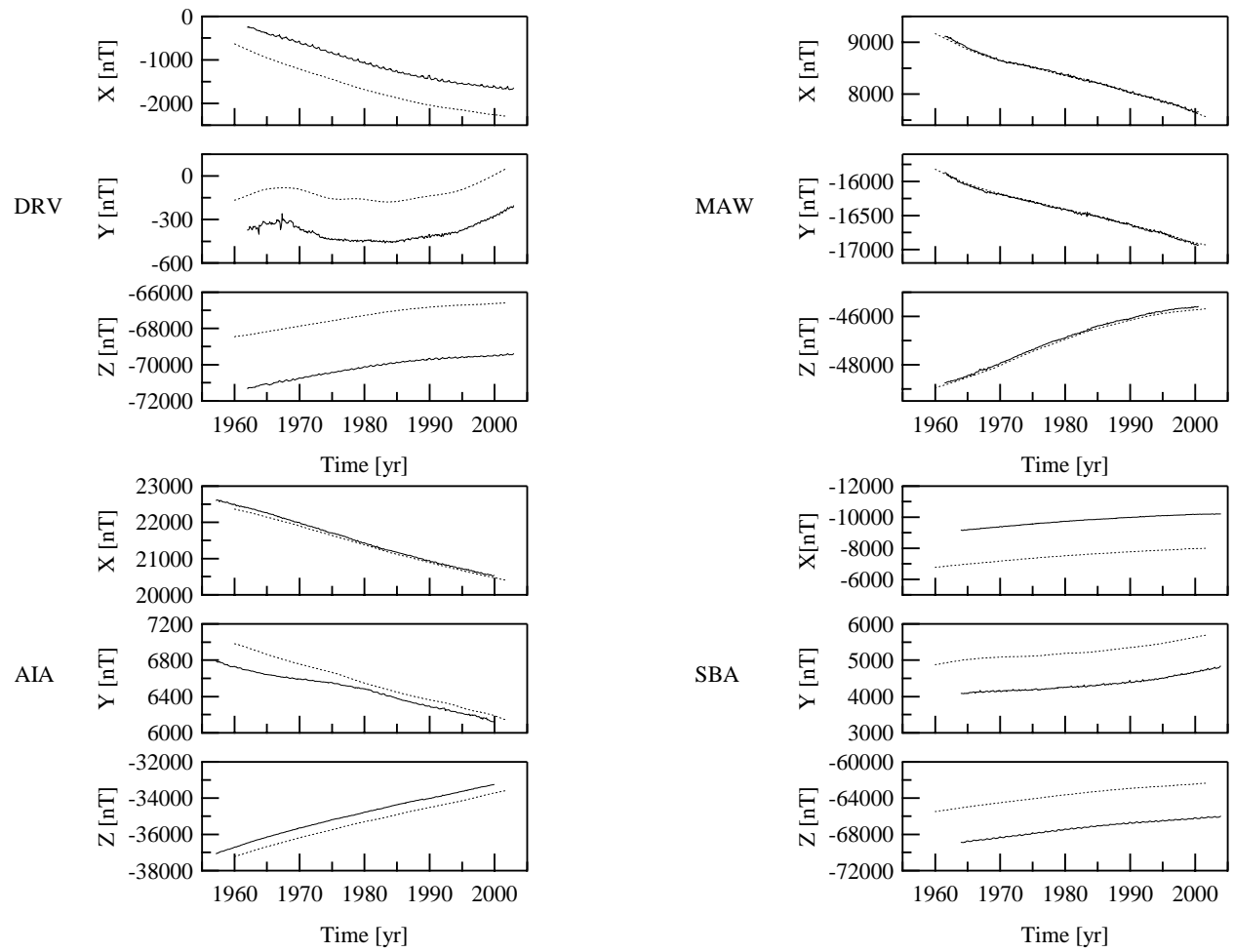

MAW
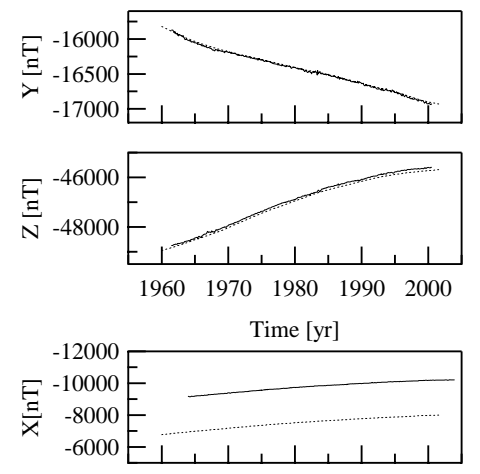

SBA

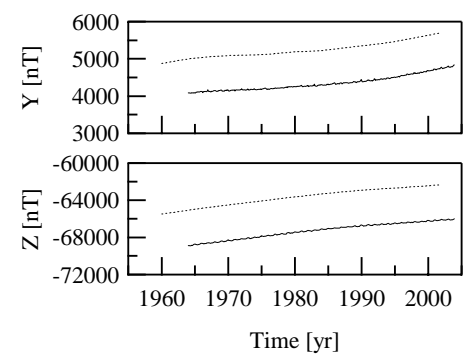

Figure 1. Comparisons between real (solid line) and synthetic (dashed line) monthly means for four observatories (AIA: lat.-65.20 ${ }^{\circ}$, long. $295.70^{\circ}$; DRV: lat. $-66.66^{\circ}$, long. $140.01^{\circ}$; MAW: lat. $-67.60^{\circ}$, long. $62.88^{\circ}$; SBA: lat. $-77.85^{\circ}$, long. $\left.166.78^{\circ}\right)$.
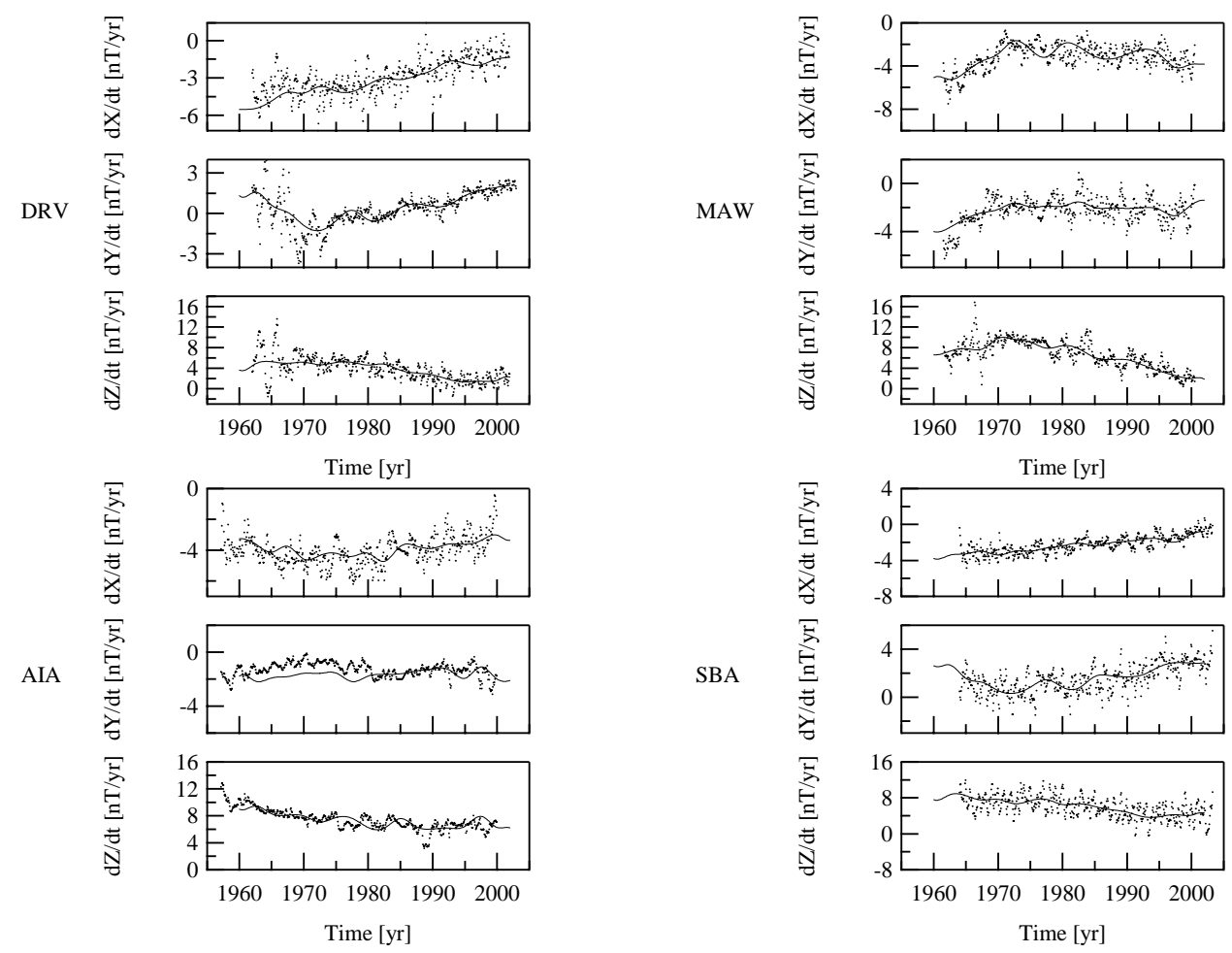

Figure 2. Monthly means for $\mathrm{X}, \mathrm{Y}$ and $\mathrm{Z}$ secular variation components (dots: real data; solid line: synthetic data given by CM4 model) for the same geomagnetic observatories reported in Figure 1. 
Indeed, the comprehensive modeling approach (assuming that it represents and isolates properly the different contributions to the magnetic field) permits us to obtain secular variation signals free from time-varying external fields and their corresponding induced counterparts, as well as the spatial biases of the observatory distribution.

Available observatory monthly means for X, Y, and Z field components are compared with the synthetic means estimated from CM4 for the observatory locations. CM4 permits us to obtain the local $\mathrm{X}, \mathrm{Y}$ and $\mathrm{Z}$ magnetic field components relative only to the core field from 1960 to mid-2002.

Figure 1 compares real and synthetic means for four Antarctic observatories denoted by their IAGA codes (AIA, DRV, MAW and SBA). Some nearly constant differences appear in the synthetic values relative to the real ones. They are due to crustal biases (Mandea and Langlais, 2002), as only the core field at the observatory locations is computed with the model. In Figure 2 we compare the secular variation obtained for the same four observatories using the synthetic data given by CM4 model and the real ones that have been smoothed with a simple 12-month running average to remove mostly the external disturbance fields. The secular variation comparison shows how CM4 is able to remove the high level of scatter in the real data from non-secular causes. Note that the observatory data used actual monthly means defined as the average over all days of the month and all times of the day, whereas the synthetic data were evaluated during magnetically quiet periods. In the synthetic data, all the contributions coming from magnetospheric and ionospheric fields, which are particularly intense in polar regions, were removed as well as the high level of noise that characterizes the Antarctic observatories.

Usually geomagnetic jerks are particularly visible in the $\mathrm{Y}$ component, which is supposed to be the least affected by the external fields. However, for the Antarctic region, the occurrence of geomagnetic jerks in the single observatories is not so clear (see Meloni et al., 2006). Nevertheless, the definition of the geomagnetic jerk as a sudden change in the slope of the secular variation corresponds to a jump in the secular acceleration. This suggests that if we analyze a time sequence of secular acceleration maps over the Antarctic region, geomagnetic jerks will be temporally located when jumps in secular acceleration values take place. In this work we have focused on the three so-called events of 1969, 1978 and 1991. Using the CM4 model, we analyzed the secular acceleration maps relative to the $\mathrm{Y}$ component over the Antarctic region at different epochs. These time intervals were selected taking into account the highly asymmetric geographical distribution of geomagnetic jerks (Alexandrescu et al, 1996; De Michelis and Tozzi, 2005).
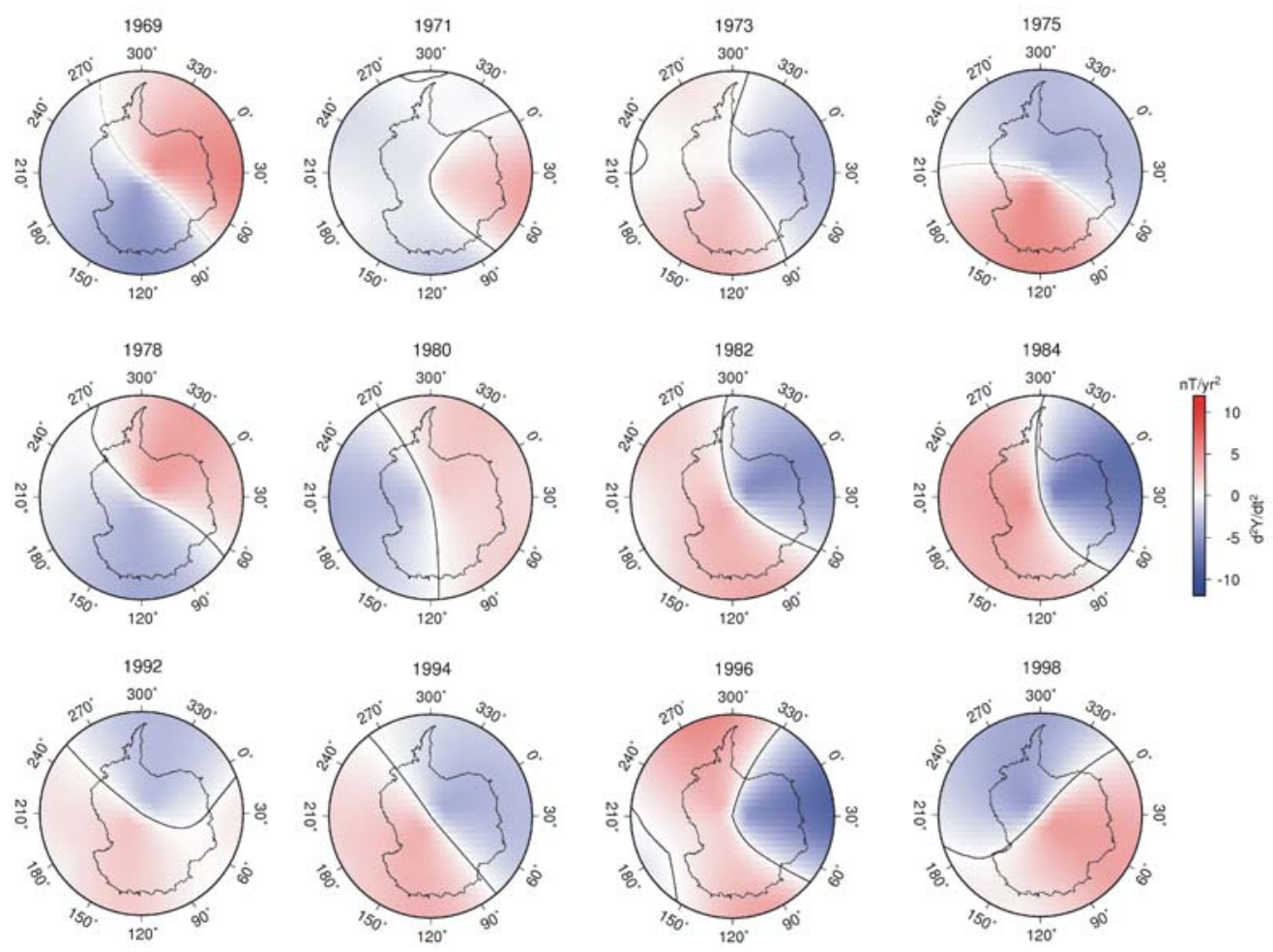

Figure 3. Secular acceleration maps relative to the Y component over the Antarctic continent obtained using synthetic data given by the CM4 model. The solid line corresponds to the zero separating the zone characterized by negative (blue) and positive (red) values of secular acceleration. 
Indeed, the three geomagnetic jerks (1969, 1978 and 1991) are characterized by prominent lags in their occurrences between the Northern and Southern Hemispheres. In the Southern Hemisphere, for example, the geomagnetic jerk usually occurs with a time lag of a few years relative to the Northern Hemisphere. For this reason we have analyzed secular acceleration maps relative to the Y component from 1969 to 1975 to look for the occurrence of the so-called 1969 jerk, from 1978 to 1984 for the 1978 jerk, and from 1992 to 1998 for the 1991 jerk. The secular acceleration maps obtained using data given by the CM4 model are reported in Figure 3. The abrupt change of color (blue-red, red-blue) of the secular acceleration maps suggests that the 1969 jerk occurred in the Antarctic region around 1972, the 1978 jerk around 1981, and the 1991 event around 1997.

\section{Summary and conclusions}

The knowledge of the fundamental features of the geomagnetic field in Antarctica is probably even more important than elsewhere in the world, because of the strong and rapid fluctuations of magnetic elements in this area. However, measurements of the Earth's magnetic field in the Antarctic continent date back only to the IGY (1957-1958). Thus, the geomagnetic observatory records from this region provide only a limited 50-year contribution. Mathematical models (e.g. IGRF, see MacMillan and Maus, 2005) are usually used to make world maps of the Earth's magnetic field and of its secular variation. These models based on the international co-operation among geomagnetic field data contributors and modelers are good for understanding the principal properties of the magnetic field in the region hosting the observatories. However, regions such as the Antarctic continent or the Pacific Ocean generally lack effective distributions of permanent ground-based observatories. Geomagnetic field behavior in these areas may be effectively studied using the CM4 model, which was derived from observatory data as well as data from satellite missions. CM4 is able to separate the different sources that contribute to the magnetic field variations observed on the Earth's surface. We used this model for a search of geomagnetic jerks over the Antarctic continent studying the secular acceleration maps in time. This approach provides information on sudden changes in the secular variation that is difficult or impossible to obtain from the data of single permanent Antarctic ground-based observatory. Geomagnetic data coming from the Antarctic region are indeed characterized by high levels both of noise and disturbances produced by electric currents flowing in the magnetosphere and ionosphere. For this reason, it is not always easy to detect the presence of geomagnetic jerks in these data. The use of secular acceleration maps permitted us to overcome this problem and to date the occurrence of the global jerks at the end of the 20th century in the Antarctic continent.
Acknowledgements. The authors would like to thank the co-editor Fred Davey and two anonymous referees for their contribution that helped to improve the manuscript.

\section{References}

Alexandrescu, M., D. Gibert, G. Hulot, J.L. Le Mouël, and G. Saracco (1996), Worldwide wavelet analysis of geomagnetic jerks, J. Geophys. Res., 101, 21975-21994.

Bloxham, J., S. Zatman and M. Dumberry (2002), The origin of geomagnetic jerks, Nature, 420, 65-68.

Chambodut, A, and M. Mandea (2005), Evidence for geomagnetic jerks in comprehensive models, Earth Planets Space, 57, 139-149.

Chambodut, A, M. Mandea, and C. Eymin (2005), Geomagnetic jerks detected from comprehensive magnetic field models, Geophys. Res. Abstracts, 7, 05165.

Courtillot, V., J. Ducruix, and J.L. Le Mouël (1978), Sur une accélération récente de la variation séculaire du champ magnétique terrestre, C.R. Acad. Sci. Ser. D, 287, 1095-1098.

De Michelis, P., L. Cafarella, and A. Meloni (2000), A global analysis of the 1991 geomagnetic jerk, Geophys. J. Int., 143, 545-556.

De Michelis, P., and R. Tozzi (2005), A local intermittency measure (LIM) approach to the detection of geomagnetic jerks, Earth Planet. Sci. Lett., 235, 261-272.

De Santis, A., R. Tozzi, and L. Gaya-Piqué (2004), Information Content and K-entropy of the Present Earth Magnetic Field, Earth Planet. Sci. Lett., 218, 269-275.

Gubbins, D., A. L. Jones, and C.C. Finlay (2006), Fall in Earth's magnetic field is erratic, Science, 312, 900-902.

Kerridge, D. (2001), Intermagnet: worldwide near-real-time geomagnetic observatory data, Proceedings of the Workshop on Space Weather, ESTEC.

Lepidi, S., L. Cafarella, P. Francia, A. Meloni, P. Palangio, and J. J. Schott (2003), Low frequency geomagnetic field variations at Dome C (Antarctica), Annales Geophysicae, 21, 923-932.

Macmillan, S. (1996), A geomagnetic jerk for the early 1990’s, Earth Planet. Sci. Lett., 137, 189-192.

Malin, S.R.C., and B.M. Hodder (1982), Was the 1970 geomagnetic jerk of internal or external origin?, Nature, 296, 726-728.

MacMillan, S. and S. Maus (2005), International Geomagnetic Reference Field - the tenth generation, Earth Planets and Space, 57, 1135-1140.

Mandea, M., E. Bellanger, and J.L. Le Mouël (2000), A geomagnetic jerk for the end of the $20^{\text {th }}$ century?, Earth Planet. Sci. Lett., 183, 369373.

Mandea, M., and B. Langlais (2002), Observatory crustal magnetic biases during MAGSAT and Ørsted satellite missions, Geophys. Res. Lett., 29, 10.1029/2001GL013693.

Meloni, A., L. R. Gaya-Piqué, P. De Michelis, and A. De Santis (2006), Some recent characteristics of geomagnetic secular variations in Antarctica, from: Fütterer D.K. at al. (eds) Antarctica: Contributions to global earth sciences, Spring-Verlag, Berlin Heidelberg New York, 377-382.

Nagao, H., T. Iyemori, T. Higuchi, and T. Araki (2003), Lower mantle conductivity anomalies estimated from geomagnetic jerks, J. Geophys. Res., 108, 2254, doi: 10.1029/2002JB001786.

Olsen, N., and M. Mandea (2007), Investigation of a secular variation impulse using satellite data: the 2003 geomagnetic jerk, Earth Planet. Sci. Lett., 255, 94-105.

Rajaram, G., T. Arun, A. Dhar, and G. Patil (2002), Rapid decrease in total magnetic field $\mathrm{F}$ at Antarctic stations - its relationship to coremantle features, Antarctic Sci., 14, 61-68.

Sabaka, T., N. Olsen, and M. E. Purucker (2004), Extending comprehensive models of the Earth's magnetic field with Ørsted and CHAMP data, Geophys. J. Int., 159, 521-547.

Waddington, R., D. Gubbins, and N. Barber (1995), Geomagnetic field analysis-V. Determining steady core-surface flows directly from geomagnetic observations, Geophys. J. Int., 122, 326-350. 